\title{
Fault Reconstruction of Islanded Nonlinear DC Microgrids: An LPV-based Sliding Mode Observer Approach
}

Asadi, Samira; Vafamand, Navid; Moallem, Mehrdad; Dragicevic, Tomislav

Published in:

IEEE Journal of Emerging and Selected Topics in Power Electronics

Link to article, DOI:

10.1109/JESTPE.2020.3043491

Publication date:

2021

Document Version

Peer reviewed version

Link back to DTU Orbit

Citation (APA):

Asadi, S., Vafamand, N., Moallem, M., \& Dragicevic, T. (2021). Fault Reconstruction of Islanded Nonlinear DC Microgrids: An LPV-based Sliding Mode Observer Approach. IEEE Journal of Emerging and Selected Topics in Power Electronics, 9(4), 4606 - 4614. https://doi.org/10.1109/JESTPE.2020.3043491

\section{General rights}

Copyright and moral rights for the publications made accessible in the public portal are retained by the authors and/or other copyright owners and it is a condition of accessing publications that users recognise and abide by the legal requirements associated with these rights.

- Users may download and print one copy of any publication from the public portal for the purpose of private study or research.

- You may not further distribute the material or use it for any profit-making activity or commercial gain

- You may freely distribute the URL identifying the publication in the public portal 


\title{
Fault Reconstruction of Islanded Nonlinear DC Microgrids: An LPV-based Sliding Mode Observer Approach
}

\author{
Samira Asadi ${ }^{1}$, Navid Vafamand ${ }^{1}$, Mehrdad Moallem ${ }^{2}$, Member, IEEE and Tomislav Dragičević ${ }^{3}$, \\ Senior Member, IEEE \\ ${ }^{1}$ School of Electrical and Computer Engineering, Shiraz University, Shiraz, Iran. \\ ${ }^{2}$ School of Mechatronic Systems Engineering, Simon Fraser University, British Columbia, Canada. \\ ${ }^{3}$ Department of Electrical Engineering, Technical University of Denmark, Lyngby, Denmark.
}

\begin{abstract}
This paper investigates the problem of fault detection and reconstruction in direct current microgrids (DC MGs) with nonlinear loads. First, a state-space representation of the DC MG with nonlinear constant power loads (CPLs) is obtained. The faults in components, actuators, and sensors in a DC MG system are modeled by using additive terms in the state-space and measurement equations. A novel robust nonlinear detector is then proposed to estimate and reconstruct the faults. The proposed detector deploys a sliding mode technique and a polytopic linear parameter varying (LPV) approach. The proposed approach utilizes the robustness and simplicity offered by the sliding mode and polytopic LPV approaches to provide a simple, but effective, detector. By employing the Lyapunov stability theory, sufficient detector design conditions are derived in terms of linear matrix inequalities (LMIs) which are numerically solved by convex optimization techniques. The proposed approach is experimentally tested on a practical DC MG benchmark connected to a CPL. Furthermore, the results are compared with other state-of-the-art methods.
\end{abstract}

Index Terms - DC microgrid, fault detection and reconstruction, constant power load, polytopic linear parameter varying (LPV) model, sliding mode detector, linear matrix inequality (LMI).

\section{INTRODUCTION}

Microgrids (MGs) have been introduced to provide an effective way of integrating various distributed renewable energy sources [1]. The MGs are classified into DC and AC ones. There has been a great deal of research on AC MGs, mainly due to its compatibility with traditional AC electrical power systems. On the other hand, in applications involving renewable DC sources (e.g., wind and photovoltaics) and DC electronic loads, the DC MGs are more suitable and costeffective than conventional AC MGs [1]. Due to avoiding frequency control, regulation of the DC MGs is simpler than AC MGs. However, there is a growing share of loads which are tightly controlled by power converters in DC MGs. Such loads are nonlinear since they act as CPLs. In the small-signal sense, they exhibit negative incremental resistance which may destabilize the overall system. In recent years, the stability issue of the CPLs in the DC MGs has been widely investigated with several control methods proposed such as backstepping [2], robust linear [3], fuzzy-model-based [4], (linear parameter varying) LPV-based reset [5], and model predictive control [6]. However, in these control approaches, it is assumed that the overall system operates without any faults. Indeed, a literature search indicates that the fault operation of islanded DC MGs that feed CPLs has been rarely investigated. On the other hand, rigorous faults may damage the MG's components if they are not identified and compensated properly [7]. The faults can originate either from the dysfunctionality of individual components or connections of the conductors to ground and/or each other. These faults can also be categorized as steady-state and transient. In [8], different faults were considered and applied to a DC MG with multiple CPLs, and a robust faulttolerant control (FTC) method was suggested. However, the aforementioned approach uses a fault-tolerant controller for each of the CPLs, which is not cost-effective. In [9], [10], the effect of sensor fault on a typical DC MG was investigated. In [8]-[10], a robust controller or monitoring technique was presented to mitigate the effect of the occurring faults; however, the faults were not reconstructed. In [11], the above methods were classified as passive FTC methods, in which the controller can guarantee the reliability of systems in the presence of weak faults with limited amplitudes. In the active FTC techniques, the information about faults is required. Such information is then exploited in the controller to actively mitigate faults. The fault information is obtained by a fault detection and isolation (FDI) scheme. This fact illustrates the importance of applying FDI techniques to practical systems [9]. The main objectives of the FDI are to initially produce an alarm when a fault is detected, followed by diagnosing the faults by determining their location and structure [13].

Among the existing FDI methods, observer-based ones have attracted a great deal of attention due to their effectiveness and theoretical merits [14]. The rationale behind observer-based FDI is to estimate both the states and faults of a system based on the available measurements [13]. Sliding mode observer (SMO) is one of the most effective and robust observer-based FDI methods, which aims at estimating the actuator and sensor faults. The superiority of the SMOs is their resiliency against uncertainties and external perturbations [12]. In [15], a linear 
state-space representation was obtained and an SMO was developed for AC and DC MGs. However, CPLs in MGs would introduce significant nonlinearities. Thereby, a nonlinear SMO (NSMO) is required. Since NSMOs have a complex design procedure in nonlinear systems, the combination of conventional NSMOs and polytopic LPV models is presented in this paper as a solution that can offer a systematic scheme for the FDI issue.

Recently, several papers have exploited the LPV-based NSMOs as a tool for the detection and isolation of faults in continuous-time systems, in which the time-varying parameters are assumed to be measurable [16]-[19]. Other works extend the results to the systems with unmeasurable time-varying parameters [20], [21]. In [16]-[20], a quadratic Lyapunov function (QLF) was proposed to investigate the stability of the system. These approaches escalate the conservative nature of FDI methods and thereby degrade the FDI transient and steadystate performances, or may lead to unfeasible results.

In this paper, a systematic and highly effective approach for FDI of DC MGs with nonlinear CPLs is proposed. The presented approach is based on the NSMO, the polytopic LPV model, and a parameter-dependent Lyapunov function (PDLF). The main contributions of this paper are as follows. First, the proposed approach can reconstruct additive actuator and sensor faults. To the best knowledge of the authors, this paper is the first attempt to study and reconstruct the occurring faults of nonlinear stand-alone DC MGs feeding CPLs, which is one of the major contributions of this paper. Furthermore, utilizing the PDLF method provides less conservative LMI conditions and brings about faster and more accurate fault reconstructions. The proposed approach is applicable to a wide range of nonlinear systems which can be represented by a polytopic LPV representation with unmeasurable premise variables. The polytopic LPV approach has certain advantages over other nonlinear methods. First, its design procedure is simple and mainly based on the linear systems theory. Secondly, it offers a systematic approach to design an observer. Moreover, the observer gains are computed via LMI techniques. Finally, an experimental DC MG with one CPL is considered as a case study. The merits of the proposed FDI method over existing methods are illustrated by applying them to the case study and comparing the obtained results.

This paper is organized as follows: Section II presents the class of DC MG with CPLs. Section III presents the derivation of the polytopic-LPV model of DC MG. In Section IV, the proposed NSMO approach is discussed. Section V presents experimental results and conclusions are presented in Section VI.

\section{DC MICROGRID DYNAMICS}

A simplified circuit diagram of the DC MG considered in this paper comprises of $\mathrm{AC}$ and/or DC sources, constant power loads (CPLs), energy storage units, and RLC filters. This structure of DC MGs is shown in Fig. 1, which is widely utilized in several applications including more electric aircraft, ships, and automotive systems [3], [22].

Since the goal of this paper is to address the FDI issue, only one DC source is considered. Nevertheless, the proposed approach is applicable to DC MGs with several sources. An overall state-space representation of the DC MG with one DC source, one energy storage system (ESS), and Q CPLs, as shown in Fig. 2, is obtained as follows [3]

$$
\dot{x}=A x+D H+B_{e s} i_{e s}+B_{s} V_{d c}
$$

where $x=\left[\begin{array}{lllll}x_{1}^{T} & x_{2}^{T} & \ldots & x_{Q}^{T} & x_{s}\end{array}\right]^{T}, x_{j}=\left[\begin{array}{ll}i_{L, j} & v_{C, j}\end{array}\right]^{T}$ is the state vector of the $j$-th CPL filter comprises the current of the $j$-the inductor and the voltage of the $j$-the capacitor, respectively, $x_{S}=\left[\begin{array}{ll}i_{L, S} & v_{C, S}\end{array}\right]^{T}, i_{e s}$ is the control input of system associated with the ESS, and $V_{d c}$ is the voltage of the DC source. Also, $H=\left[h_{1}, \ldots, h_{Q}\right]^{T}$ with $h_{j}=\frac{1}{v_{C, j}}$ and

$$
\begin{gathered}
A=\left[\begin{array}{ccccc}
A_{1} & 0 & \cdots & 0 & A_{1 s} \\
0 & A_{2} & \cdots & 0 & A_{2 s} \\
\vdots & \vdots & \ddots & \vdots & \vdots \\
0 & 0 & \cdots & A_{Q} & A_{Q s} \\
A_{c n} & A_{c n} & \cdots & A_{c n} & A_{s}
\end{array}\right], B_{e s}=\left[\begin{array}{c}
0 \\
\vdots \\
0 \\
b_{e s}
\end{array}\right], \\
D=\left[\begin{array}{cccc}
d_{1} & 0 & \cdots & 0 \\
0 & d_{2} & \cdots & 0 \\
\vdots & \vdots & \ddots & \vdots \\
0 & 0 & \cdots & d_{Q} \\
0 & 0 & \cdots & 0
\end{array}\right], B_{s}=\left[\begin{array}{c}
0 \\
\vdots \\
0 \\
b_{s}
\end{array}\right], d_{j}=\left[\begin{array}{c}
0 \\
\frac{P_{j}}{C_{j}}
\end{array}\right], \\
A_{j}=\left[\begin{array}{ccc}
-\frac{r_{j}}{L_{j}} & -\frac{1}{L_{j}} \\
\frac{1}{C_{j}} & 0
\end{array}\right], A_{j s}=\left[\begin{array}{cc}
0 & \frac{1}{L_{j}} \\
0 & 0
\end{array}\right], b_{e s}=\left[\begin{array}{c}
0 \\
-\frac{1}{C_{s}}
\end{array}\right], \\
A_{s}=\left[\begin{array}{cc}
-\frac{r_{s}}{L_{s}} & -\frac{1}{L_{s}} \\
\frac{1}{C_{s}} & 0
\end{array}\right], b_{s}=\left[\begin{array}{c}
1 \\
L_{s} \\
0
\end{array}\right], A_{c n}=\left[\begin{array}{cc}
0 & 0 \\
-1 & 0 \\
C_{s} & 0
\end{array}\right],
\end{gathered}
$$

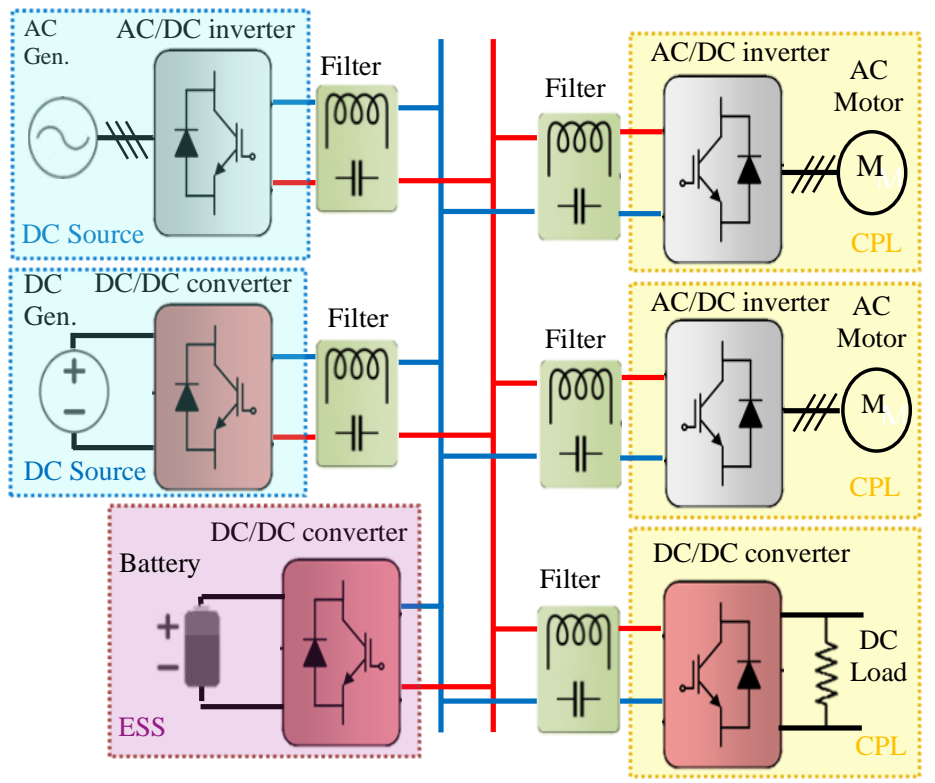

Fig. 1. Detailed block diagram of a DC MG.

The state-space representation (1) contains both linear (i.e. $A x+B_{e s} i_{e s}+B_{s} V_{d c}$ ) and nonlinear (i.e. $D H$ ) terms. Thereby, the overall dynamics are nonlinear. In the following sections, the goal is to design an LPV-based SMO for the nonlinear DC MG system (1).

\section{POLYTOPIC LPV MODELING}


A polytopic quasi-LPV model is a precise nonlinear convex aggregation of some local linear representations of the nonlinear plant [5], [20]. The convex theory facilitates deploying the well-known linear control theories to stabilize nonlinear smooth systems. To derive a polytopic quasi-LPV system, it is necessary to model the nonlinearities of (1) by its corresponding polytopic LPV representation [23]. As can be seen in (1) and (2), the overall DC MG system has $Q$ nonlinear terms (i.e. $h_{j}$ ). Without loss of generality, the equivalent polytopic quasi-LPV model for a DC MG with one CPL is obtained as [6]

$$
\dot{\tilde{x}}=\sum_{i=1}^{2} \rho_{i}\left\{A_{i} \tilde{x}+B_{e s} \tilde{i}_{e s}+B_{s} \tilde{V}_{d c}\right\}
$$

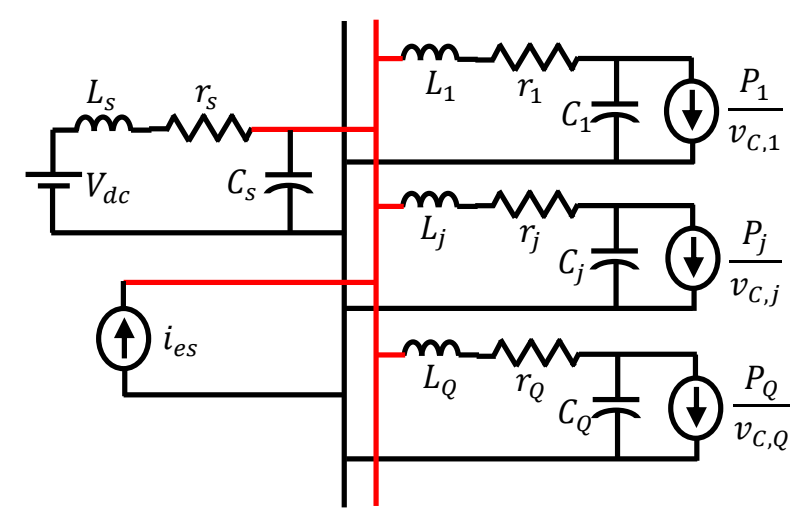

Fig. 2. Electrical schematic of the considered DC MG case study.

where $\tilde{x}, \tilde{i}_{e s}$, and $\tilde{V}_{d c}$ are the changes of coordinates of $x, i_{e s}$, and $V_{d c}$, respectively [6], and

$$
\begin{gathered}
\rho_{1}=\frac{U_{\max } \tilde{v}_{C, 1}-h_{1}}{\left(U_{\max }-U_{\min }\right) \tilde{v}_{C, 1}}, \rho_{2}=\frac{h_{1}-U_{\min } \tilde{v}_{C, 1}}{\left(U_{\max }-U_{\min }\right) \tilde{v}_{C, 1}} \\
U_{\text {min }}=\frac{\frac{1}{v_{C 0,1}\left(v_{C 0,1}+\tilde{v}_{C, 1}\right)}, U_{\max }=\frac{1}{v_{C 0,1}\left(v_{C 0,1}-\tilde{v}_{C, 1}\right)}}{A_{1}}=\left[\begin{array}{cccc}
-\frac{r_{1}}{L_{1}} & -\frac{1}{L_{1}} & 0 & \frac{1}{L_{1}} \\
\frac{1}{C_{1}} & \frac{P_{1}}{C_{1}} U_{\min } & 0 & 0 \\
0 & 0 & -\frac{r_{s}}{L_{s}} & -\frac{1}{L_{s}} \\
-\frac{1}{C_{s}} & 0 & \frac{1}{C_{s}} & 0
\end{array}\right], B_{e s}=\left[\begin{array}{c}
0 \\
0 \\
0 \\
-\frac{1}{C_{s}}
\end{array}\right], \\
A_{2}=\left[\begin{array}{cccc}
-\frac{r_{1}}{L_{1}} & -\frac{1}{L_{1}} & 0 & \frac{1}{L_{1}} \\
\frac{1}{C_{1}} & \frac{P_{1}}{C_{1}} U_{\text {max }} & 0 & 0 \\
0 & 0 & -\frac{r_{s}}{L_{s}} & -\frac{1}{L_{s}} \\
-\frac{1}{C_{s}} & 0 & \frac{1}{C_{s}} & 0
\end{array}\right], B_{s}=\left[\begin{array}{c}
0 \\
0 \\
\frac{1}{L_{s}} \\
0
\end{array}\right]
\end{gathered}
$$

where the time-varying parameters $\rho_{1}$ and $\rho_{2}$ are functions of system states and assure the convexity constraint. Thereby, the LPV model (3) is called the polytopic quasi-LPV. Besides, the obtained polytopic LPV model is valid for the given region $-\widetilde{w}_{2,1} \leq \widetilde{v}_{C, 1} \leq \widetilde{w}_{2,1}$. The details of polytopic LPV modeling of DC MGs with CPLs can be found in [5]. The goal is to reconstruct the occurring faults in (3). Therefore, such a system is merged with actuator and sensor faults and disturbance as

$$
\left\{\begin{array}{l}
\dot{\tilde{x}}=\sum_{i=1}^{r} \rho_{i}\left\{A_{i} \tilde{x}+B_{e s} \tilde{i}_{e s}+B_{s} \tilde{V}_{d c}+M_{i} f_{a}+D_{i} \xi\right\} \\
y=C \tilde{x}+N f_{s}
\end{array}\right.
$$

where $f_{a} \in R^{q}, f_{s} \in R^{h}, \xi \in R^{l}, \tilde{x} \in R^{n}$, and $y \in R^{p}$ are unknown actuator and sensor faults, the system disturbance, ,the measured outputs and the state vector, respectively. Also, $M_{i} \in R^{n \times q}, D_{i} \in R^{n \times l}, C \in R^{p \times n}$, and $N \in R^{p \times h}$ are constant real matrices.

\section{MAIN RESUlT}

First, the system dynamics (5) is re-written in a way that all faults appear in the dynamics and the new output vector does not contain any faults. To perform this, a proper orthogonal matrix $T_{r} \in R^{p \times p}$ is derived by using the $\mathrm{QR}$ reduction of matrix $N$ [12], and the new state vector is constructed as $X=$ $\left[\begin{array}{ll}x^{T} & \mu^{T}\end{array}\right]^{T} \in R^{n+h}$ where $\mu \in R^{h}$ is defined as follows:

$$
\dot{\mu}=-A_{f} \mu+A_{f} C_{2} x+A_{f} N_{2} f_{s}
$$

Thus, the new polytopic-LPV system of order $n+h$ is given by

$$
\begin{aligned}
& \left\{\begin{array}{l}
\dot{X}=\sum_{i=1}^{r} \hat{\rho}_{i}\left\{\mathcal{A}_{i} X+\mathcal{B}_{e s} \tilde{l}_{e s}+\mathcal{B}_{s} \tilde{V}_{d c}+\mathcal{M}_{i} f+\mathcal{D}_{i} \xi+\phi\right\} \\
Y=\mathcal{C} X
\end{array}\right. \\
& \mathcal{A}_{i}=\left[\begin{array}{cc}
A_{i} & 0 \\
A_{f} C_{2} & -A_{f}
\end{array}\right], \mathcal{B}_{e s}=\left[\begin{array}{c}
B_{e s} \\
0
\end{array}\right], \mathcal{B}_{s}=\left[\begin{array}{c}
B_{s} \\
0
\end{array}\right], \\
& \mathcal{D}_{i}=\left[\begin{array}{c}
D_{i} \\
0
\end{array}\right], \mathcal{M}_{i}=\left[\begin{array}{cc}
M_{i} & 0 \\
0 & A_{f} N_{2}
\end{array}\right], \mathcal{C}=\left[\begin{array}{cc}
C_{1} & 0 \\
0 & I_{h}
\end{array}\right] \\
& \phi:=\sum_{i=1}^{r}\left(\rho_{i}-\hat{\rho}_{i}\right)\left\{\mathcal{A}_{i} X+\mathcal{M}_{i} f+\mathcal{D}_{i} \xi\right\}
\end{aligned}
$$

$f=\left[\begin{array}{ll}f_{a}^{T} & f_{s}^{T}\end{array}\right]^{T} \in R^{q+h}, \quad N_{2} \in R^{h \times h}$ is a nonsingular matrix and $A_{f}>0 \in R^{h \times h}$ is a user-defined matrix with positive eigenvalues. The time-varying parameters $\hat{\rho}_{i}$ are based on the state estimation vector $\hat{x}$. Also, $\phi$ satisfies the Lipschitz condition with a positive scalar $\gamma$, as follows:

$$
\|\phi\| \leq \gamma\|x-\hat{x}\| . \quad \forall x, \hat{x} \in R^{n}
$$

Some assumptions and lemmas are presented below to propose an NSMO for the system modeled in (7).

$$
\begin{aligned}
& \text { Assumptions: } \\
& \operatorname{rank}\left(\mathcal{C}\left[\mathcal{M}_{i} \mathcal{D}_{i}\right]\right)=q+h+l \\
& \operatorname{rank}\left[\begin{array}{ccc}
S I-\mathcal{A}_{i} & \mathcal{M}_{i} & \mathcal{D}_{i} \\
\mathcal{C} & 0 & 0
\end{array}\right]=n+2 h+q+l \text { for } \\
& \operatorname{Re}(s) \geq 0 \quad n+h>p \geq q+h+l \\
& \quad\|f\| \leq \rho_{f} ; \quad\|\xi\| \leq \rho_{d} ; \quad\left\|\dot{\rho}_{k}\right\| \leq \rho_{m k}
\end{aligned}
$$

Lemma 1 [12]: If (10) holds, changes of coordinates $\bar{T}_{i}$ can be found so that the triples $\left(\mathcal{A}_{i},\left[\mathcal{M}_{i} \mathcal{D}_{i}\right], \mathcal{C}\right)$ are of the following format:

$$
\begin{gathered}
\mathcal{A}_{i}=\left[\begin{array}{ll}
\mathcal{A}_{11 . i} & \mathcal{A}_{12 . i} \\
\mathcal{A}_{21, i} & \mathcal{A}_{22 . i}
\end{array}\right], \mathcal{A}_{21, i}=\left[\begin{array}{l}
\mathcal{A}_{211, i} \\
\mathcal{A}_{212, i}
\end{array}\right], \\
{\left[\mathcal{M}_{i} \mathcal{D}_{i}\right]=\left[\begin{array}{cc}
0 & 0 \\
\mathcal{M}_{2 . i} & \mathcal{D}_{2 . i}
\end{array}\right], \mathcal{C}=\left[\begin{array}{ll}
0 & \mathrm{~T}_{0}
\end{array}\right]}
\end{gathered}
$$


where $\quad \mathcal{A}_{11 . i} \in R^{(n+h-p) \times(n+h-p)}, \quad \mathcal{A}_{211 . i} \in$ $R^{(p-q-h-l) \times(n+h-p)}, \mathrm{T}_{0} \in R^{p \times p}$ is an orthogonal matrix, and $\mathcal{M}_{2 . i} \in R^{p \times(q+h)}$ and $\mathcal{D}_{2 . i} \in R^{p \times l}$ have the following format:

$$
\left[\mathcal{M}_{2 . i} \mathcal{D}_{2 . i}\right]=\left[\begin{array}{cc}
0 & 0 \\
\mathcal{M}_{0 . i} & \mathcal{D}_{0 . i}
\end{array}\right]
$$

Note that $\left[\mathcal{M}_{0 . i} \mathcal{D}_{0 . i}\right] \in R^{(q+h+l) \times(q+h+l)}$ is nonsingular.

Lemma 2 [24]: The parameter-dependent inequality $\sum_{i=1}^{r} \sum_{j=1}^{r} \rho_{i} \rho_{j} Q_{i j}<0$ holds if the following parameterindependent inequalities hold:

$$
\begin{cases}Q_{i i}<0 & \text { for } i=1, \ldots, r \\ \frac{2}{1-r} Q_{i i}+Q_{i j}+Q_{j i}<0 & \text { for } i \neq j=1, \ldots, r\end{cases}
$$

The proposed NSMO is defined based on the coordinate transformation $\bar{T}_{i}$ given in Lemma 1 , as follows

$$
\left\{\begin{array}{l}
\dot{\hat{X}}=\sum_{i=1}^{r} \hat{\rho}_{i}\left\{\begin{array}{c}
\mathcal{A}_{i} \hat{X}+\mathcal{B}_{e s} \tilde{i}_{e s}+\mathcal{B}_{s} \tilde{V}_{d c}+G_{l, i} e_{y}+ \\
\eta_{f, i} G_{n, i} \operatorname{sign}\left(e_{Y}\right)+\eta_{d, i} G_{d, i} \operatorname{sign}\left(e_{Y}\right)
\end{array}\right\} \\
\hat{Y}=\mathcal{C} \hat{X}
\end{array}\right.
$$

where $e_{Y}:=Y-\hat{Y}$ is the output error estimation and is considered as the sliding surface, in this paper. Thereby, the estimation vector $X$ of the NSMO is modified by the terms $\eta_{f, i} G_{n, i} \operatorname{sign}\left(e_{Y}\right)+\eta_{d, i} G_{d, i} \operatorname{sign}\left(e_{Y}\right)$. The scalars $\eta_{f, i}$ and $\eta_{d, i}$ satisfy the following inequalities

$$
\begin{aligned}
& \eta_{f, i} \geq \rho_{f}\left\|T_{0} M_{2, i}\right\| \max _{j}\left(\frac{\left\|P_{2, j}\right\|}{\lambda_{\min }\left(P_{2, j}\right)}\right)+w_{f, i} \\
& \eta_{d, i} \geq \rho_{d}\left\|T_{0} \mathcal{N}\right\| \max _{j}\left(\frac{\left\|P_{2, j}\right\|}{\lambda_{\min }\left(P_{2, j}\right)}\right)+w_{d, i} \\
& 1, \ldots, r
\end{aligned}
$$

with $w_{f, i}$ and $w_{d, i}$ are arbitrary positive constants. Also, the sign function operator is defined as follows:

$$
\operatorname{sign}\left(e_{Y}\right)=\left\{\begin{array}{lc}
\frac{e_{Y}}{\left\|e_{Y}\right\|} & e_{Y} \neq 0 \\
0 & \text { otherwise }
\end{array}\right.
$$

Moreover, the design matrices $G_{n . i}$ and $G_{l . i}$ should be chosen to assure the stability of the tracking error dynamics. This issue will be discussed in Theorem 1. Before presenting Theorem 1, the state estimation error $e:=X-\hat{X}$, and its dynamics are derived by considering (7) and (17), as follows

$$
\dot{e}=\sum_{i=1}^{r} \widehat{\rho}_{i}\left\{\begin{array}{c}
\left(\mathcal{A}_{i}-G_{l . i} \mathcal{C}\right) e+\mathcal{M}_{i} f+\mathcal{D}_{i} \xi- \\
\eta_{f, i} G_{n, i} \operatorname{sign}\left(e_{Y}\right)-\eta_{d, i} G_{d, i} \operatorname{sign}\left(e_{Y}\right)+\phi
\end{array}\right\}
$$

Now define another change of coordinates as follows

$$
T_{L . i}=\left[\begin{array}{cc}
I_{n+h-p} & L_{i} \\
0 & T_{0}
\end{array}\right], L_{i}=\left[\begin{array}{ll}
\bar{L}_{i} & 0
\end{array}\right] \in R^{(n+h-p) \times p}
$$

where $\bar{L}_{i} \in R^{(n+h-p) \times(p-q-h-l)}$ is the stabilizing gain matrix, the system (20) can be re-written as

$$
\dot{\tilde{e}}=\left[\begin{array}{c}
\dot{e}_{1} \\
\dot{e}_{Y}
\end{array}\right]=\sum_{i=1}^{r} \hat{\rho}_{i}\left\{\begin{array}{c}
\mathcal{A}_{\mathrm{t}, i} \tilde{e}+T_{L, i} \phi+\tilde{\mathcal{M}}_{i} f- \\
\eta_{f, i} \tilde{G}_{n . i} \operatorname{sign}\left(e_{Y}\right)+ \\
\widetilde{\mathcal{D}}_{i} \xi-\eta_{d, i} \tilde{G}_{n . i} \operatorname{sign}\left(e_{Y}\right)
\end{array}\right\}
$$

where

$$
\mathcal{A}_{\mathrm{t}, i}=\left[\begin{array}{cc}
\mathcal{A}_{11 . \mathrm{i}}+L_{i} \mathcal{A}_{21 . \mathrm{i}} & 0 \\
T_{0} \mathcal{A}_{21 . \mathrm{i}} & \mathcal{A}_{\text {s.i }}
\end{array}\right]
$$

in which $\mathcal{A}_{s . i}$ is a user-defined matrix with negative eigenvalues. Moreover, the matrix $L_{i}(21)$ is selected such that $\mathcal{A}_{11 . \mathrm{i}}+L_{i} \mathcal{A}_{21 . \mathrm{i}}<0$. Since $\mathcal{A}_{\mathrm{t}, i}$ has a zero off-diagonal matrix, its eigenvalues are the same as its diagonal matrix elements. As a consequence, since $\mathcal{A}_{11 . \mathrm{i}}+L_{i} \mathcal{A}_{21 . \mathrm{i}}$ and $\mathcal{A}_{\text {s.i }}$ have negative eigenvalues, the matrix $\mathcal{A}_{\mathrm{t}, i}(23)$ is asymptotically stable.

In the following Theorem 1 , the sufficient LMI conditions, which guarantee the asymptotic stability of (22) with a maximized admissible Lipschitz constant $\gamma^{*}$ for the $\phi$ in (9), will be derived.

Theorem 1. If for the given scalars $\varepsilon>0$, and $\sigma>0$, there exist matrices $P_{1 . i}$ and $P_{2 . i}$ such that the following LMI optimization problem has a solution

$$
\min (\sigma+\varepsilon)
$$

Subject to

$$
\begin{gathered}
P_{1 . i}=P_{1 . i}^{T}>0 \\
P_{2 . i}=P_{2 . i}^{T}>0 \\
\left\{\begin{array}{lr}
Q_{i i}<0 & \text { for } i=1, \ldots, r \\
\frac{2}{1-r} Q_{i i}+Q_{i j}+Q_{j i}<0 & \text { for } i \neq j=1, \ldots, r
\end{array}\right. \\
\left\{\begin{array}{lr}
Q_{i i}{ }^{\prime}<0 & \text { for } i=1, \ldots, r \\
\frac{2}{1-r} Q_{i i}{ }^{\prime}+Q_{i j}{ }^{\prime}+Q_{j i}{ }^{\prime}<0 & \text { for } i \neq j=1, \ldots, r
\end{array}\right.
\end{gathered}
$$

where

$$
\begin{aligned}
& Q_{i j}= {\left[\begin{array}{ccccccc}
\Delta_{1 . i j} & \mathcal{A}_{21 . i}{ }^{T} T_{0}{ }^{T} P_{2 . j} & P_{1 . j} & 0 & I & 0 & \mathcal{A}_{21 . i}{ }^{T} \\
* & \Delta_{2 . i j} & 0 & P_{2 . j} & 0 & I & 0 \\
* & * & -\varepsilon I & 0 & 0 & 0 & 0 \\
* & * & * & -\varepsilon I & 0 & 0 & 0 \\
* & * & * & * & -\sigma I & 0 & 0 \\
* & * & * & * & * & -\sigma I & 0 \\
* & * & * & * & * & * & -\beta I
\end{array}\right] } \\
& Q_{i j}{ }^{\prime}= {\left[\begin{array}{ccccccc}
\Delta_{1 . i j} & \mathcal{A}_{21 . i}{ }^{T} T_{0}{ }^{T} P_{2 . j} & P_{1 . j} & 0 & I & 0 & P_{1 . j} \\
* & \Delta_{2 . i j} & 0 & P_{2 . j} & 0 & I & 0 \\
* & * & -\varepsilon I & 0 & 0 & 0 & 0 \\
* & * & * & -\varepsilon I & 0 & 0 & 0 \\
* & * & * & * & -\sigma I & 0 & 0 \\
* & * & * & * & -\sigma I & 0 \\
* & * & * & * & * & -\beta I
\end{array}\right] } \\
& \Delta_{1 . i j}=\mathcal{A}_{11 . i}{ }^{T} P_{1 . j}+P_{1 . j} \mathcal{A}_{11 . i}+\sum_{k=1}^{r} \rho_{m k} P_{1 . k} \\
& \Delta_{2 . i j}=\mathcal{A}_{\mathrm{s} . i \mathrm{i}}{ }^{T} P_{2 . j}+P_{2 . j} \mathcal{A}_{\mathrm{s.i}}+\sum_{k=1}^{r} \rho_{m k} P_{2 . k}
\end{aligned}
$$

with $\rho_{m k}$ defined in (13). Finally, the estimation error is bounded and the maximized admissible Lipschitz constant is $\gamma^{*}=\max (\gamma)=\frac{1}{\left\|T_{L}\right\|\left\|T_{L}{ }^{-1}\right\| \sqrt{\varepsilon \sigma}}$.

Proof: See the proof in Appendix A.

Remark 1- Lipchitz constraint: In this paper, the Lipchitz constraint (9) is utilized to deal with the nonlinearity term $\phi$. The Lipchitz condition is a simple but effective way to convert nonlinear terms into quasi-linear ones and facilitate deriving the controller/observer design conditions in terms of LMIs [25], [26]. As can be seen in (39) of Appendix A, the asymptotic stability of error dynamics is achieved regardless the value of the Lipchitz constant $\gamma$. However, the value of $\gamma$ affects the local region of attraction (ROA). In [27], the effect of the Lipchitz constraint on the ROA for DC MGs systems with CPLs is studied. furthermore, a robust stabilizing controller is suggested and its sufficient design conditions are derived in 
terms of LMIs. Also, in [28] a Kalman-based robust linear controller for the same power system is developed.

Since the sliding surface reaches zero, on the sliding surface $e_{Y}$ one concludes $e_{Y}=\dot{e_{Y}}=0$. Consequently, the actuator fault, sensor fault, and the disturbance input are reconstructed as follows

$$
\begin{gathered}
{\left[\begin{array}{l}
\hat{f}_{a} \\
\hat{f}_{s}
\end{array}\right]=\left(\sum_{k=1}^{r} \hat{\rho}_{i}\left\{\mathcal{M}_{2 . i}\right\}\right)^{\dagger} T_{0}{ }^{-1} \sum_{k=1}^{r} \hat{\rho}_{i}\left\{\eta_{f . i} \operatorname{sign}\left(e_{Y}\right)\right\},} \\
\hat{\xi}=\left(\sum_{k=1}^{r} \hat{\rho}_{i}\left\{\left[\mathcal{D}_{2 . i}\right\}\right)^{\dagger} T_{0}{ }^{-1} \sum_{k=1}^{r} \hat{\rho}_{i}\left\{\eta_{d . i} \operatorname{sign}\left(e_{Y}\right)\right\}\right.
\end{gathered}
$$

To reduce the chattering of the constructed signals, the sign function is replaced by the saturation one, as follows

$$
\begin{gathered}
{\left[\begin{array}{l}
\hat{f}_{a} \\
\hat{f}_{s}
\end{array}\right]=\left(\sum_{k=1}^{r} \hat{\rho}_{i}\left\{\mathcal{M}_{2 . i}\right\}\right)^{\dagger} T_{0}{ }^{-1} \sum_{k=1}^{r} \hat{\rho}_{i}\left\{\eta_{f . i} \operatorname{sat}\left(e_{Y}\right)\right\},} \\
\hat{\xi}=\left(\sum_{k=1}^{r} \hat{\rho}_{i}\left\{\left[\mathcal{D}_{2 . i}\right\}\right)^{\dagger} T_{0}{ }^{-1} \sum_{k=1}^{r} \hat{\rho}_{i}\left\{\eta_{\text {d.i }} \operatorname{sat}\left(e_{Y}\right)\right\}\right.
\end{gathered}
$$

where $\dagger$ represents the pseudo-inverse of a matrix and

$$
\operatorname{sat}\left(e_{Y}\right)=\frac{e_{Y}}{\left\|e_{Y}\right\|+\delta_{f}}
$$

with $\delta_{a}$ and $\delta_{s}$ are small positive user-defined constants.

Remark 2- Overall design procedure: The main steps to numerically solve Theorem 1 can be summarized as follows:

Step 1. Find the transfer matrix $T_{r} \in R^{p \times p}$ by using the QR reduction of matrix $N$ and obtain the transformed system (7).

Step 2. Check the assumptions (10)-(13).

Step 3. Find the changes of coordinates $\bar{T}_{i}$ such that the triples $\left(\mathcal{A}_{i},\left[\mathcal{M}_{i} \mathcal{D}_{i}\right], \mathcal{C}\right)$ are in the format $(14)$.

Step 4. Compute the scalars $\sigma$ and $\varepsilon$ and the matrices $L_{i}$ from the LMIs (24)-(28).

Step 5. Compute the matrices $T_{L . i}$ from the obtained matrices $L_{i}$ based on (21).

Step 6. Compute the maximized admissible Lipschitz constant which is $\gamma^{*}=\max (\gamma)=\frac{1}{\left\|T_{L}\right\|\left\|T_{L}{ }^{-1}\right\| \sqrt{\varepsilon \sigma}}$.

Step 7. Reconstruct the faults and disturbance by using equations (30) and (31).

Remark 3- Merits of the proposed approach and its design challenges: Almost all prior researches on fault detection in nonlinear systems, represented by polytopic LPV systems through SMOs, have deployed a QLF (Quadratic Lyapunov function) [16]-[20]. The novelty of the proposed fault detection approach is reducing the conservativeness of the state-of-the-art methods by employing a PDLF (ParameterDependent Lyapunov Function), which reduces the conservativeness of LMI conditions. The proposed approach is established based on LPV systems. So, it detects and constructs sensor and actuator faults of nonlinear systems. Since, LPV model and PDLF are utilized, conventional change of variables presented in [16]-[20] are not useful. Thereby, the Finsler's lemma is utilized. Also, since the PDLF comprises several Lyapunov matrices, the structure of the sliding mode fault observer is adopted. On the other hand, this paper focuses on the issue of fault detection and reconstruction for DC MGs with CPLs for the first time. In this paper, a proper approach is presented to effectively detect and reconstruct the DC MGs with CPLs plant faults, simultaneously.
V. REAL-TIME SIMULATION AND EXPERIMENTAL RESULTS

To evaluate the performance advantages including fast transient response and robustness characteristics, the developed NSMO is tested on a DC MG characterized in Table I, and the results are compared with [29]-[31]. The parameters in Table I are chosen such that the DC MG is stable and, thereby, no power from the ESS to the DC MG is injected (i.e. $\tilde{i}_{e s}=0$ ). Moreover, the matrices are chosen as follows to meet the conditions (10)-(12).

$$
\begin{gathered}
C=\left[\begin{array}{llll}
1 & 0 & 0 & 0 \\
0 & 1 & 0 & 0 \\
0 & 0 & 1 & 1
\end{array}\right], D_{1}=D_{2}=\left[\begin{array}{llll}
0 & 1 & 1 & 0
\end{array}\right]^{T}, \\
M_{1}=M_{2}=\left[\begin{array}{lll}
1 & 1 & 1-1
\end{array}\right]^{T}, N=\left[\begin{array}{lll}
1 & 0 & 1
\end{array}\right]^{T}
\end{gathered}
$$

Using Theorem 1, the feasible results are obtained as $\varepsilon=$ $0.3651, \sigma=0.3653, \gamma^{*}=2.7379$.

Scenario 1. Real-time software-in-the-loop simulations: In the first scenario, the proposed approach is compared with the EKF method of [29] and the TS fuzzy approach [30]. Although in [29], the issue of fault detection is not investigated, the presented approach is used to estimate unknown CPL powers. Utilizing the above approach, it is possible to detect actuator faults by augmenting them (instead of CPL power) in the system states and estimating them via the joint-EKF of [29]. The initial covariance matrices of the joint-EKF are set as $R=$ $10^{-4} I^{3}$ and $Q=10^{-4} I^{5}$, where $I^{b}$ is a $b$-dimensional identity matrix. Also, in the TS fuzzy approach [30], the actuator fault and disturbance are estimated. To have a fair comparison, only the actuator fault is considered in this scenario. To perform the real-time simulations, all proposed NSMO, joint-EKF algorithm [29], and TS fuzzy approach [30] are implemented by the OPAL-RT hardware, connected to a personal computer with the DK60 board. Figs. 3(a)-(e) represent the estimation errors of the states and actuator fault. As can be seen in Fig. 3, although the proposed approach estimates the system states with a small ultimate bound, the actuator fault is constructed more accurately than the joint-EKF algorithm [29] and TS fuzzy approach [30]. Note that the steady-state estimation error is due to using the saturation function, instead of the sign function, in (30). On the other hand, the joint-EKF algorithm [29] and the TS fuzzy approach [30] are not able to estimate the actuator and sensor faults and disturbance, simultaneously.

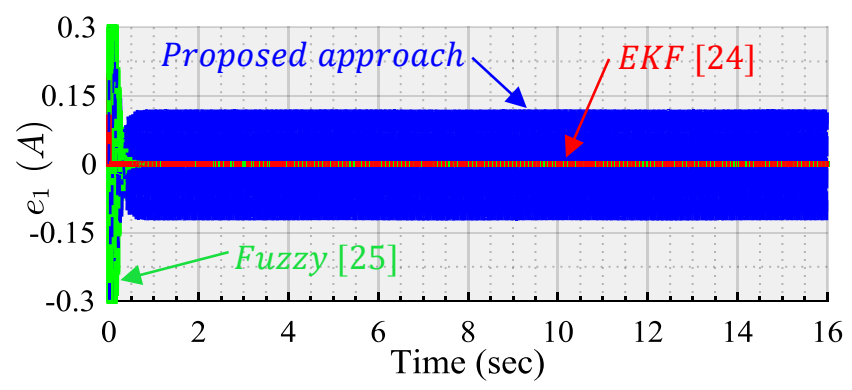

(a). 


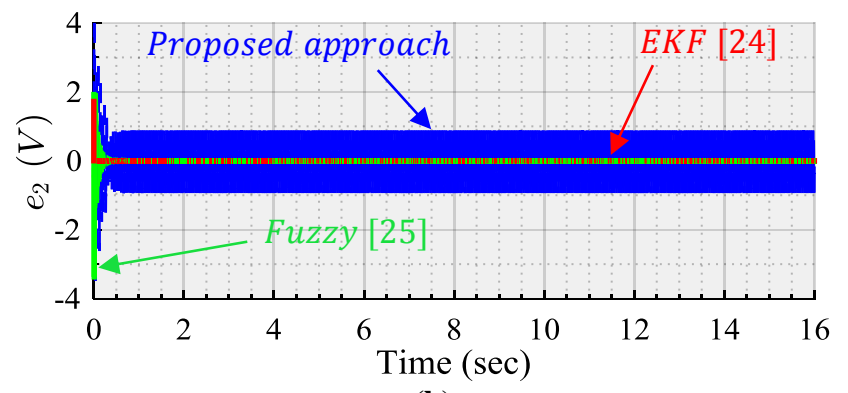

(b).

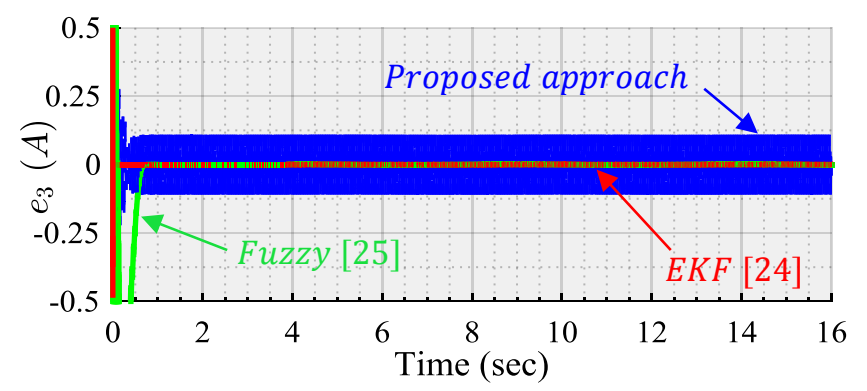

(c).

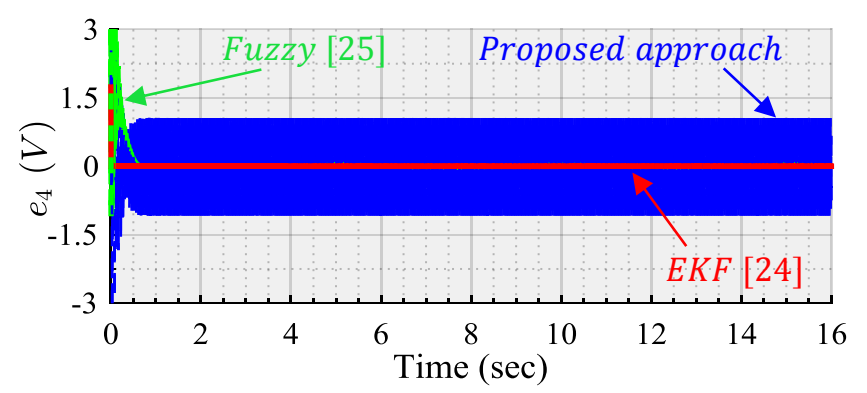

(d).

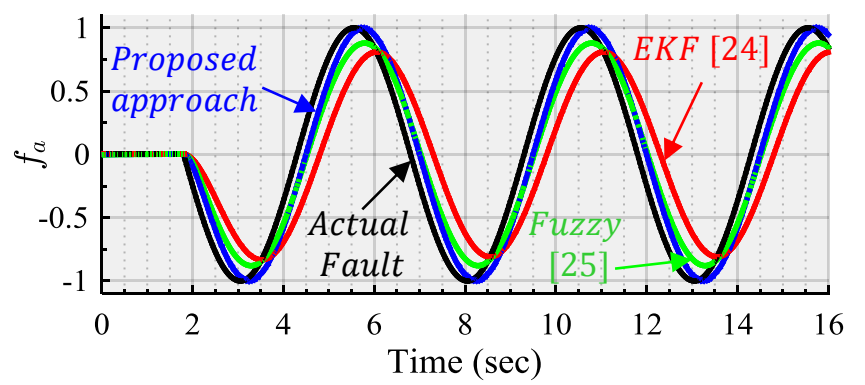

(e).

Fig. 3. Faults and disturbance introduced in the DC MG: (a)

Estimation error of $i_{L, 1}$, (b) Estimation error of $v_{C, 1}$, (c) Estimation error of $i_{L, S}$, (d) Estimation error of $v_{C, S},(\mathbf{e})$ Actuator fault.

Table I: Parameters for DC MG with one CPL

\begin{tabular}{ccc}
\hline \hline$r_{1}=1.1 \Omega$ & $P_{1}=300 \mathrm{~W}$ & $C_{s}=500 \mu \mathrm{F}$ \\
$L_{1}=4 \mathrm{mH}$ & $\tilde{x}_{2 N}=130.4$ & $L_{s}=4 \mathrm{mH}$ \\
$C_{1}=500 \mu \mathrm{F}$ & $r_{s}=1.1 \Omega$ & $V_{d c}=200 \mathrm{~V}$ \\
\hline \hline
\end{tabular}

Furthermore, the re-construction error specifications of the actuator fault are given in Table II. As can be seen in Table II, one observes that the fault estimation accuracy of the proposed approach remarkable outperforms the other approaches in the sense of norms 2 and $\infty$, mean absolute error (MAE) and mean square error (MSE).
Table II: Error specifications of the actuator fault for all approaches

\begin{tabular}{|c|c|c|c|}
\hline Approach & $\begin{array}{c}\text { Proposed } \\
\text { Approach }\end{array}$ & EKF [29] & Fuzzy [30] \\
\hline Error $L_{2}$ norm & 63.6023 & 152.6336 & 128.5209 \\
Error $L_{\infty}$ norm & 0.2457 & 0.5912 & 0.3943 \\
MAE & 0.1340 & 0.3231 & 0.2756 \\
MSE & 0.0253 & 0.1456 & 0.1032 \\
\hline
\end{tabular}

Scenario 2. Experimental results: The proposed NSMO approach [31] is verified for an experimental set-up equivalent to the dynamics (1). The OPAL-RT is used as a real-time Simulator (RTS) which monitors the DC MG and estimates the faults. The experimental DC MG setup and its simplified schematic are drawn in Fig. 4. Moreover, the QLF-based approach [31] is considered for the comparison. The FDI method [31] can construct actuator and sensor faults for a disturbed system.

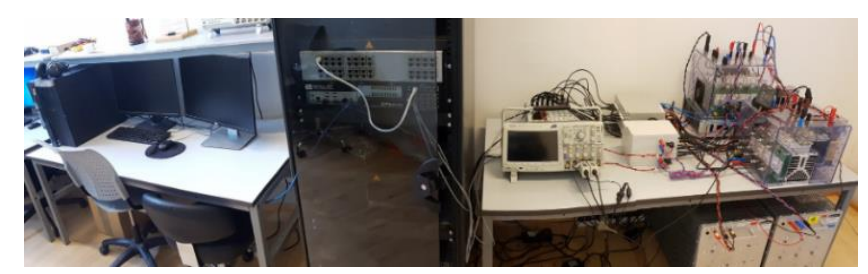

(a).

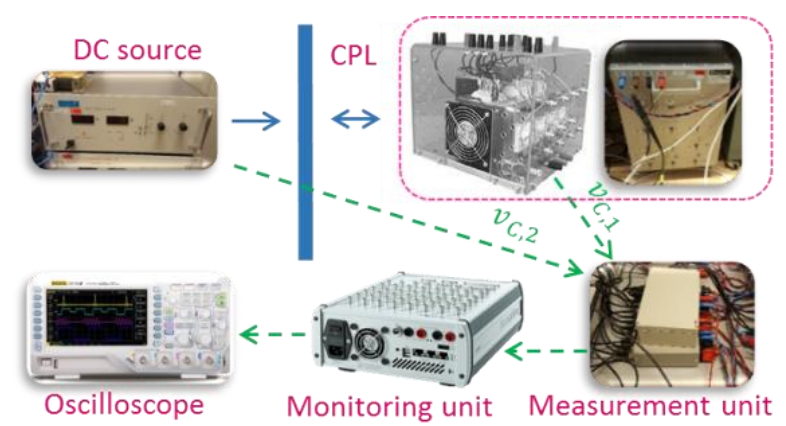

(b).

Fig. 4. Experimental setup of the DC MG: (a) Overall setup, (b) Simplified schematic.

Figs. 5(a)-(c) demonstrate that the proposed LPV-based NSMO approach is able to reconstruct the disturbance input and the occurring faults more efficiency in comparison with the QLF method presented in [31].

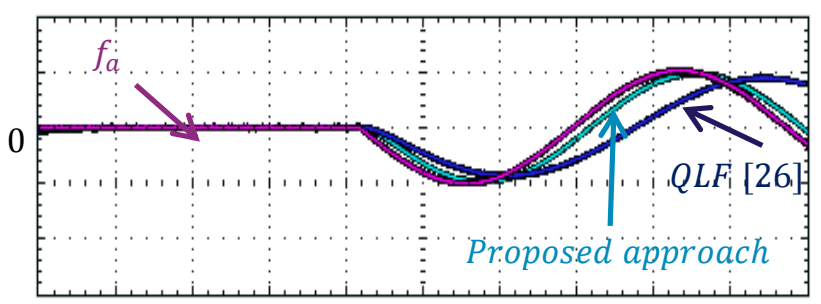

(a). 

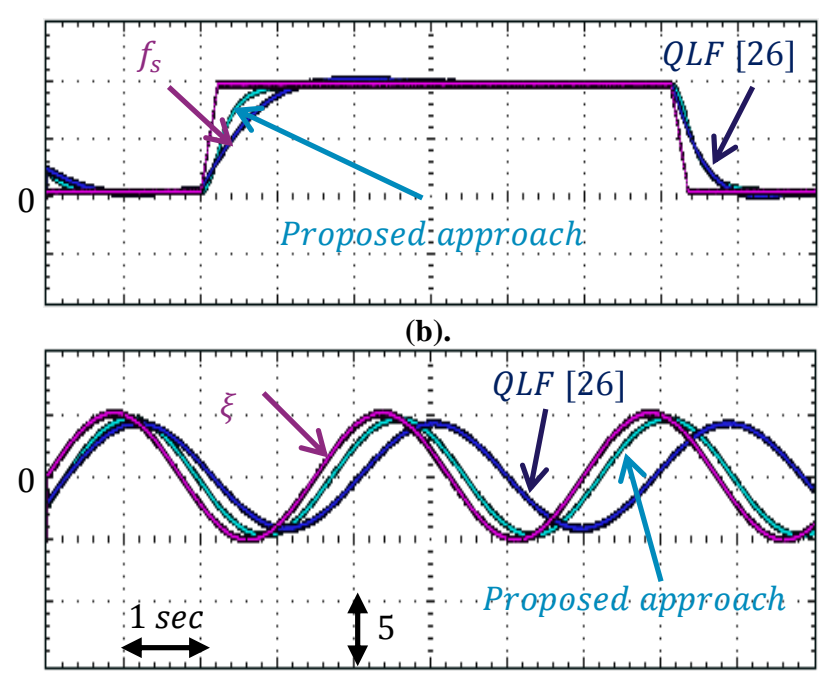

(c).

Fig. 5. Faults and disturbances introduced in the DC MG: (a) Actuator fault, (b) Sensor fault, (c) Disturbance.

Fig. 6 shows the estimation error of the system states. As it can be observed, the state estimation errors converge to zero in finite-time. Moreover, both the proposed approach and QLFbased approach [31] lead to a small state estimation error because they use NSMO with saturation functions.

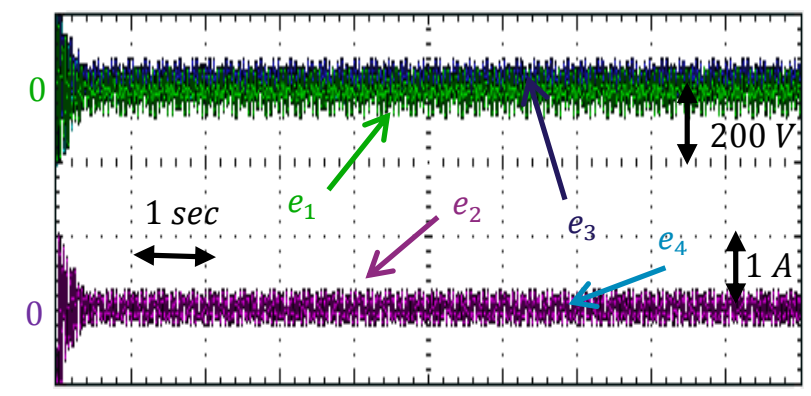

Fig. 6. State estimation errors.

\section{CONCLUSION}

In this paper, the problem of fault detection and reconstruction in DC MGs with nonlinear loads was investigated. A novel robust gain-scheduling detector was established based on a sliding mode technique and a polytopic quasi-LPV approach was proposed to estimate and construct the occurring faults of a DC MG. The proposed approach was tested for robustness in face of disturbances. The results indicate that the proposed method can effectively estimate the system states and construct the system faults. Experimental results on a DC MG with one CPL demonstrate that the proposed approach was able to estimate the constant and timevarying faults very fast with small oscillations. Also, the estimated faults and disturbances are more accurate than other methods in the literature. For future work, further analysis of the effects of the faults on the DC system to locate the faults is of great importance.

\section{APPENDIX A}

The following parameter-dependent Lyapunov function (PDLF) is employed to verify the asymptotic stability of (22) and the performance of the observer (17), as follows

$$
V=\tilde{e}^{T}\left(\sum_{j=1}^{r} \hat{\rho}_{i} P_{j}\right) \tilde{e}
$$

where $\quad P_{j}=\operatorname{diag}\left(P_{1 . j}, P_{2 . j}\right) \quad$ with $\quad P_{1 . j}=P_{1 . j}^{T}>0 \in$ $R^{(n+h-p) \times(n+h-p)}$ and $P_{2 . j}=P_{2 . j}^{T}>0 \in R^{p \times p}$ are Lyapunov candidate matrices. The Lyapunov function (32) is called (PDLF) because it comprises the time-varying scheduling parameters. The time derivative of the PDLF along with the dynamics (22) is given by

$$
\begin{gathered}
\dot{V}=\sum_{i=1}^{r} \sum_{j=1}^{r} \hat{\rho}_{i} \hat{\rho}_{j}\left\{\tilde{e}^{T}\left(\mathcal{A}_{\mathrm{t} . i}{ }^{T} P_{j}+P_{j} \mathcal{A}_{\mathrm{t} . i}+\sum_{k=1}^{r} \dot{\hat{\rho}}_{k} P_{k}\right) \tilde{e}\right. \\
+2 \tilde{e}^{T} P_{j}\left(\tilde{\mathcal{M}}_{i} f-\eta_{f, i} \tilde{G}_{n . i} \operatorname{sign}\left(e_{Y}\right)+\widetilde{\mathcal{D}}_{i} \xi\right. \\
\left.\left.-\eta_{d, i} \tilde{G}_{n . i} \operatorname{sign}\left(e_{Y}\right)+T_{L} \phi\right)\right\}
\end{gathered}
$$

By using (9) and considering the fact that $2 \psi^{T} \vartheta \leq \frac{1}{\varepsilon} \psi^{T} \psi+\varepsilon \vartheta^{T} \vartheta$ with $\varepsilon>0$, it follows that

$$
2 \tilde{e}^{T} P_{j} T_{L} \phi \leq \frac{1}{\varepsilon} \tilde{e}^{T} P_{j} P_{j} \tilde{e}+\varepsilon \alpha^{2}\|\tilde{e}\|^{2}
$$

where $\alpha=\left\|T_{L}\right\|\left\|T_{L}^{-1}\right\| \gamma$. From (13), we obtain

$$
\begin{aligned}
& \tilde{e}^{T} P_{j}\left(\tilde{\mathcal{M}}_{i} f-\eta_{f, i} \tilde{G}_{n . i} \operatorname{sign}\left(e_{Y}\right)\right) \\
& \leq-w_{f . i} \lambda_{\min }\left(P_{2 . j}\right)\left\|e_{Y}\right\| \leq 0
\end{aligned}
$$

and

$$
\begin{aligned}
& \tilde{e}^{T} P_{j}\left(\widetilde{\mathcal{D}}_{i} \xi-\eta_{d, i} \tilde{G}_{n . i} \operatorname{sign}\left(e_{Y}\right)\right) \\
& \quad \leq-w_{d . i} \lambda_{\min }\left(P_{2 . j}\right)\left\|e_{Y}\right\| \leq 0
\end{aligned}
$$

Using (34)-(36), the (33) can be written as

$$
\begin{gathered}
\dot{V} \leq \sum_{i=1}^{r} \sum_{j=1}^{r} \hat{\rho}_{i} \hat{\rho}_{j}\left\{\tilde { e } ^ { T } \left(\mathcal{A}_{\mathrm{t} . i}{ }^{T} P_{j}+P_{j} \mathcal{A}_{\mathrm{t} . i}+\varepsilon^{-1} P_{j} P_{j}\right.\right. \\
\left.\left.+\sigma^{-1} I_{n+h}+\sum_{k=1}^{r} \dot{\hat{\rho}}_{k} P_{k}\right) \tilde{e}\right\}
\end{gathered}
$$

where $\sigma:=\left(\varepsilon \alpha^{2}\right)^{-1}$. From (13), we obtain

$$
\tilde{e}^{T} \sum_{k=1}^{r} \dot{\hat{\rho}}_{k} P_{k} \tilde{e} \leq \tilde{e}^{T} \sum_{k=1}^{r} \rho_{m k} P_{k} \tilde{e}
$$

Thereby, (37) takes the following form

$$
\begin{aligned}
\dot{V} \leq & \sum_{i=1}^{r} \sum_{j=1}^{r} \hat{\rho}_{i} \hat{\rho}_{j} \times \\
& \left\{\left[\begin{array}{l}
e_{1} \\
e_{Y}
\end{array}\right]^{T}\left[\begin{array}{cc}
\emptyset_{1} & \mathcal{A}_{21 . i}{ }^{T} T_{0}{ }^{T} P_{2 . j} \\
P_{2 . j} T_{0} \mathcal{A}_{21 . i} & \emptyset_{2}
\end{array}\right]\left[\begin{array}{l}
e_{1} \\
e_{Y}
\end{array}\right]\right\}<0
\end{aligned}
$$

where $\quad \emptyset_{1}=P_{1 . j} \mathcal{A}_{11 . i}+P_{1 . j} L_{i} \mathcal{A}_{21 . i}+\mathcal{A}_{11 . i}^{T} P_{1 . j}+$ $\mathcal{A}_{21 . i}^{T} L_{i}^{T} P_{1 . j}+\varepsilon^{-1} P_{1 . j} P_{1 . j}+\sigma^{-1} I_{n+h-P}+\sum_{k=1}^{r} \rho_{m k} P_{1 . k}, \emptyset_{2}=$ $P_{2 . j} \mathcal{A}_{\mathrm{s} . i}+\mathcal{A}_{\mathrm{s} . i}^{T} P_{2 . j}+\varepsilon^{-1} P_{2 . j} P_{2 . j}+\sigma^{-1} I_{P}+\sum_{k=1}^{r} \rho_{m k} P_{2 . k}$

Exploiting the Congruence lemma [25], (39) is satisfied by

$$
\sum_{i=1}^{r} \sum_{j=1}^{r} \hat{\rho}_{i} \hat{\rho}_{j}\left\{\left[\begin{array}{cc}
\emptyset_{1} & \mathcal{A}_{21 . i}{ }^{T} T_{0}{ }^{T} P_{2 . j} \\
P_{2 . j} T_{0} \mathcal{A}_{21 . i} & \emptyset_{2}
\end{array}\right]\right\}<0
$$

Whereas the bilinear terms $P_{1 . j} L_{i} \mathcal{A}_{21 . i}$ and $\mathcal{A}_{21 . i}^{T} L_{i}^{T} P_{1 . j}$ appear in $\emptyset_{1}$, the constraint (40) is not in the form of LMIs. To turn (40) into LMIs, the Finsler's Lemma [24] is applied on (40). Therefore, we have

$$
\left[\begin{array}{cc}
\Delta_{1}+\beta^{-1} \mathcal{A}_{21 . i}{ }^{T} \mathcal{A}_{21 . i} & \mathcal{A}_{21 . i}{ }^{T} T_{0}{ }^{T} P_{2 . j} \\
P_{2 . j} T_{0} \mathcal{A}_{21 . i} & \emptyset_{2}
\end{array}\right]<0
$$


and

$$
\left[\begin{array}{cc}
\Delta_{1}+\beta^{-1} P_{1 . j} P_{1 . j} & \mathcal{A}_{21 . i}{ }^{T} T_{0}{ }^{T} P_{2 . j} \\
P_{2 . j} T_{0} \mathcal{A}_{21 . i} & \emptyset_{2}
\end{array}\right]<0
$$

where $\quad \Delta_{1}=P_{1 . j} \mathcal{A}_{11 . i}+\mathcal{A}_{11 . i}^{T} P_{1 . j}+\varepsilon^{-1} P_{1 . j} P_{1 . j}+$ $\sigma^{-1} I_{n+h-P}+\sum_{k=1}^{r} \rho_{m k} P_{1 . k}$. In the final stage, deploying the Schur complement [24] and employing Lemma 2, the (41) and (42) lead to LMIs (27) and (28), respectively. To compute the maximum admissible Lipchitz constant, remind the definitions of $\alpha$ and $\sigma$ (i.e. $\sigma:=\left(\varepsilon \alpha^{2}\right)^{-1}$ ). Thus, we have $\gamma=\frac{\alpha}{\left\|T_{L}\right\|\left\|T_{L}{ }^{-1}\right\|}=$ $\frac{1}{\left\|T_{L}\right\|\left\|T_{L}{ }^{-1}\right\| \sqrt{\sigma \varepsilon}}$. Minimizing the sum of $\sigma$ and $\varepsilon$ (i.e. $\min (\sigma+$ $\varepsilon)$ ), maximizes the Lipchitz constant $\gamma$. Thereby, (24) is added.

\section{REFERENCES}

[1] H. Bevrani, B. Francois, and T. Ise, Microgrid Dynamics and Control. Hoboken, NJ, USA: John Wiley \& Sons, Inc., 2017.

[2] S. Yousefizadeh, J. D. Bendtsen, N. Vafamand, M. H. Khooban, T. Dragicevic, and F. Blaabjerg, "Tracking Control for a DC Microgrid Feeding Uncertain Loads in More Electric Aircraft: Adaptive Backstepping Approach," IEEE Trans. Ind. Electron., 2018.

[3] L. Herrera, W. Zhang, and J. Wang, "Stability Analysis and Controller Design of DC Microgrids With Constant Power Loads," IEEE Trans. Smart Grid, vol. 8, no. 2, pp. 881-888, Mar. 2017, doi: 10.1109/TSG.2015.2457909.

[4] M. M. Mardani, N. Vafamand, M. H. Khooban, T. Dragicevic, and F. Blaabjerg, "Design of Quadratic D-stable Fuzzy Controller for DC Microgrids with Multiple CPLs," IEEE Trans. Ind. Electron., pp. 1-1, 2018, doi: 10.1109/TIE.2018.2851971.

[5] N. Vafamand, A. Khayatian, and M. H. Khooban, "Stabilisation and transient performance improvement of DC MGs with CPLs: non-linear reset control approach," IET Gener. Transm. Distrib., vol. 13, no. 14, pp. 3169-3176, Jul. 2019, doi: 10.1049/ietgtd.2018.6739.

[6] N. Vafamand, M. H. Khooban, T. Dragicevic, and F. Blaabjerg, "Networked Fuzzy Predictive Control of Power Buffers for Dynamic Stabilization of DC Microgrids," IEEE Trans. Ind. Electron., pp. 1-1, 2018, doi: 10.1109/TIE.2018.2826485.

[7] N. Bayati, A. Hajizadeh, and M. Soltani, "Protection in DC microgrids: a comparative review," IET Smart Grid, vol. 1, no. 3, pp. 66-75, Oct. 2018, doi: 10.1049/iet-stg.2018.0035.

[8] P. Magne, B. Nahid-Mobarakeh, and S. Pierfederici, "A design method for a fault-tolerant multi-agent stabilizing system for DC microgrids with Constant Power Loads," in 2012 IEEE Transportation Electrification Conference and Expo (ITEC), Dearborn, MI, USA, Jun. 2012, pp. 1-6, doi: 10.1109/ITEC.2012.6243469.

[9] S. Saha, T. K. Roy, M. A. Mahmud, M. E. Haque, and S. N. Islam, "Sensor fault and cyber attack resilient operation of DC microgrids," Int. J. Electr. Power Energy Syst., vol. 99, pp. 540554, Jul. 2018, doi: 10.1016/j.ijepes.2018.01.007.

[10] R. Todd and A. J. Forsyth, "DC-bus power quality for aircraft power systems during generator fault conditions," IET Electr. Syst. Transp., vol. 1, no. 3, p. 126, 2011, doi: 10.1049/ietest.2010.0056.

[11] L. Frosini, C. Harlisca, and L. Szabo, "Induction Machine Bearing Fault Detection by Means of Statistical Processing of the Stray Flux Measurement," IEEE Trans. Ind. Electron., vol. 62, no. 3, pp. 1846-1854, Mar. 2015, doi: 10.1109/TIE.2014.2361115.

[12] H. Alwi, C. Edwards, and C. Pin Tan, Fault Detection and FaultTolerant Control Using Sliding Modes. London: Springer London, 2011.
[13] I. Hwang, S. Kim, Y. Kim, and C. E. Seah, "A Survey of Fault Detection, Isolation, and Reconfiguration Methods," IEEE Trans. Control Syst. Technol., vol. 18, no. 3, pp. 636-653, May 2010, doi: 10.1109/TCST.2009.2026285.

[14] J. Yang, F. Zhu, X. Wang, and X. Bu, "Robust sliding-mode observer-based sensor fault estimation, actuator fault detection and isolation for uncertain nonlinear systems," Int. J. Control Autom. Syst., vol. 13, no. 5, pp. 1037-1046, Oct. 2015, doi: 10.1007/s12555-014-0159-4.

[15] C. Mellucci, P. P. Menon, C. Edwards, and A. Ferrara, "Secondorder sliding mode observers for fault reconstruction in power networks," IET Control Theory Appl., vol. 11, no. 16, pp. 27722782, Nov. 2017, doi: 10.1049/iet-cta.2017.0249.

[16] H. Alwi, C. Edwards, and A. Marcos, "Fault reconstruction using a LPV sliding mode observer for a class of LPV systems," $J$. Frankl. Inst., vol. 349, no. 2, pp. 510-530, Mar. 2012, doi: 10.1016/j.jfranklin.2011.06.026.

[17] H. Alwi and C. Edwards, "Robust fault reconstruction for linear parameter varying systems using sliding mode observers," Int. J. Robust Nonlinear Control, vol. 24, no. 14, pp. 1947-1968, Sep. 2014, doi: 10.1002/rnc.3009.

[18] H. Hamdi, M. Rodrigues, C. Mechmeche, and N. Benhadj Braiek, "Fault diagnosis based on sliding mode observer for LPV descriptor systems," Asian J. Control, vol. 21, no. 1, pp. 89-98, Jan. 2019, doi: 10.1002/asjc.2022.

[19] M. M. Morato, O. Sename, L. Dugard, and M. Q. Nguyen, "Fault estimation for automotive Electro-Rheological dampers: LPVbased observer approach," Control Eng. Pract., vol. 85, pp. 1122, Apr. 2019, doi: 10.1016/j.conengprac.2019.01.005.

[20] L. Chen, C. Edwards, and H. Alwi, "Sensor fault estimation using LPV sliding mode observers with erroneous scheduling parameters," Automatica, vol. 101, pp. 66-77, Mar. 2019, doi: 10.1016/j.automatica.2018.10.055.

[21] S. Asadi, A. Khayatian, M. Dehghani, N. Vafamand, and M. H. Khooban, "Robust sliding mode observer design for simultaneous fault reconstruction in perturbed Takagi-Sugeno fuzzy systems using non-quadratic stability analysis," J. Vib. Control, p. 107754631989169, Jan. 2020, doi: $10.1177 / 1077546319891693$.

[22] M. A. Kardan et al., "Improved Stabilization of Nonlinear DC Microgrids: Cubature Kalman Filter Approach," IEEE Trans. Ind. Appl., vol. 54, no. 5, pp. 5104-5112, Sep. 2018, doi: 10.1109/TIA.2018.2848959.

[23] N. Vafamand, M. H. Asemani, and A. Khayatian, "Robust L1 Observer-based Non-PDC Controller Design for Persistent Bounded Disturbed TS Fuzzy Systems," IEEE Trans. Fuzzy Syst., pp. 1-1, 2017, doi: 10.1109/TFUZZ.2017.2724018.

[24] N. Vafamand, M. H. Asemani, and A. Khayatiyan, "A robust L1 controller design for continuous-time TS systems with persistent bounded disturbance and actuator saturation," Eng. Appl. Artif. Intell., vol. 56, pp. 212-221, Nov. 2016, doi: 10.1016/j.engappai.2016.09.002.

[25] A. B. Brahim, S. Dhahri, F. B. Hmida, and A. Sellami, "An Hळ sliding mode observer for Takagi-Sugeno nonlinear systems with simultaneous actuator and sensor faults An," Int. J. Appl. Math. Comput. Sci., vol. 25, no. 3, pp. 547-559, 2015, doi: 10.1515/amcs-2015-0041.

[26] A. Ben Brahim, S. Dhahri, F. Ben Hmida, and A. Sellami, "Simultaneous Actuator and Sensor Faults Reconstruction Based on Robust Sliding Mode Observer for a Class of Nonlinear Systems," Asian J. Control, vol. 19, no. 1, pp. 362-371, Jan. 2017, doi: 10.1002/asjc.1359.

[27] L. Herrera, W. Zhang, and J. Wang, "Stability Analysis and Controller Design of DC Microgrids With Constant Power Loads," IEEE Trans. Smart Grid, vol. 8, no. 2, pp. 881-888, Mar. 2017, doi: 10.1109/TSG.2015.2457909. 
[28] M. A. Kardan et al., "Improved Stabilization of Nonlinear DC Microgrids: Cubature Kalman Filter Approach," IEEE Trans. Ind. Appl., vol. 54, no. 5, pp. 5104-5112, Sep. 2018, doi: 10.1109/TIA.2018.2848959.

[29] S. Yousefizadeh, J. D. Bendtsen, N. Vafamand, M. H. Khooban, T. Dragičević, and F. Blaabjerg, "EKF-based Predictive Stabilization of Shipboard DC Microgrids with Uncertain Timevarying Loads," J. Emerg. Sel. Top. Power Electron., 2018.

[30] S. Li, H. Wang, A. Aitouche, and N. Christov, "Sliding mode observer design for fault and disturbance estimation using Takagi-Sugeno model," Eur. J. Control, vol. 44, pp. 114-122, Nov. 2018, doi: 10.1016/j.ejcon.2018.09.006.

[31] A. Ben Brahim, S. Dhahri, F. Ben Hmida, and A. Sellami, "Simultaneous Actuator and Sensor Faults Reconstruction Based on Robust Sliding Mode Observer for a Class of Nonlinear Systems," Asian J. Control, p. n/a-n/a, Jan. 2016, doi: 10.1002/asjc.1359.

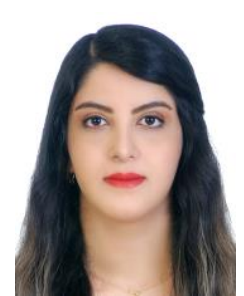

Samira Asadi was born in 1991. She received the M.S. degree in Electrical Engineering-Control from Shiraz University, Iran in 2017. She is currently a $\mathrm{PhD}$ student in Mechatronic Systems Engineering at Simon Fraser University, Canada. Her main research interests include nonlinear control systems, machine learning, and fault detection and fault tolerant control systems.

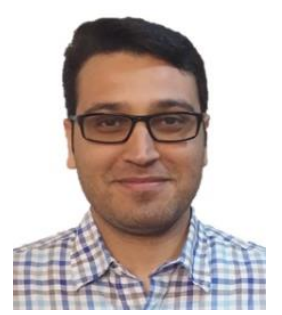

Navid Vafamand received his B.Sc. degree in electrical engineering and M.Sc. degree in control engineering form Shiraz University of Technology, Iran, in 2012 and 2014, respectively, and $\mathrm{Ph}$. D. in control engineering at Shiraz University, Shiraz, Iran, in 2019. Currently, he serves as a post doctorial researcher at Shiraz University. He was a Ph.D. Visiting student with the Department of Energy Technology, Aalborg University, Denmark, from 2017 to 2018. Dr. Vafamand is the co-author of more than 90 international conference and journal papers and two chapter-books and an active reviewer in several journals. His main research interests include Takagi-Sugeno (TS) fuzzy systems, model predictive control, dc-dc microgrids.

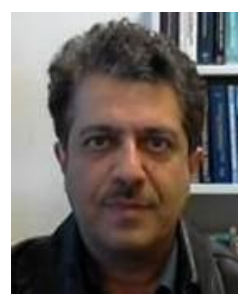

Mehrdad Moallem (S'95-M'97) received the Ph.D. degree in electrical and computer engineering from Concordia University, Montreal, QC, Canada, in 1997. From 1997 to 2007, he held research and faculty positions at Concordia University, Montreal, Canada; Duke University, Durham, NC, USA; and the University of Western Ontario, London, ON, Canada; before joining Simon Fraser University in 2007, where he is currently Professor of Mechatronic Systems Engineering. Dr. Moallem's research include multi-disciplinary areas related to control applications including mechatronics, energy systems, and power electronics. He has served on the editorial board of several conferences and journals including the American Control Conference, IEEE/ASME Transactions on Mechatronics, and IFAC Journal of Mechatronics.

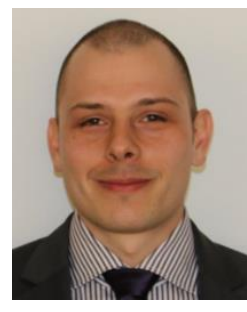

Tomislav Dragičević (Senior Member, IEEE) received the M.Sc. and the industrial Ph.D. degrees in electrical engineering from the Faculty of Electrical Engineering, University of Zagreb, Croatia, in 2009 and 2013, respectively. From 2013 to 2016, he has been a Postdoctoral Researcher with Aalborg University, Denmark, where he was an Associate Professor from 2016 to 2020. Since 2020, he has been a Professor at the Technical University of Denmark. He made a Guest Professor stay at Nottingham University, UK, during Spring/Summer of 2018. His research interests include application of advanced control, optimization and artificial intelligence inspired techniques to provide innovative and effective solutions to emerging challenges in design, control and cyber-security of power electronics intensive electrical distributions systems and microgrids. He has authored and coauthored more than 250 technical publications (more than 120 of them are published in international journals, mostly in IEEE), eight book chapters, and one book in the SE fields. He serves as an Associate Editor of the IEEE Transactions on Industrial Electronics, IEEE Transactions on Power Electronics, IEEE Emerging and Selected Topics in Power Electronics, and IEEE Industrial Electronics Magazine. He is a recipient of the Končar Prize for the Best Industrial Ph.D. Thesis in Croatia, a Robert Mayer Energy Conservation Award, and he is a winner of an Alexander von Humboldt Fellowship for Experienced Researchers. 